\title{
Articular Bone Surface
}

National Cancer Institute

\section{Source}

National Cancer Institute. Articular Bone Surface. NCI Thesaurus. Code C32143.

The bone surface in a joint. 